\title{
Efficacy, Safety, and Patient-Reported Outcomes in Patients with Moderate-to-Severe Plaque Psoriasis Treated with Brodalumab for 5 Years in a Long-Term, Open-Label, Phase II Study
}

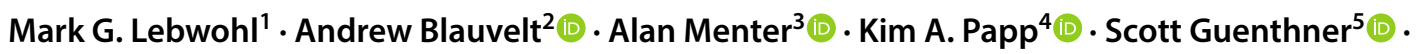 \\ Radhakrishnan Pillai ${ }^{6} \cdot$ Robert J. Israel $^{7} \cdot$ Abby Jacobson $^{8}$
}

Published online: 14 November 2019

(C) The Author(s) 2019

\begin{abstract}
Background Chronic inflammatory diseases such as psoriasis require treatment options that maintain efficacy and tolerability during extended treatment.

Objective The aim of the study was to assess the long-term efficacy and safety of brodalumab, a fully human anti-interleukin-17 receptor A monoclonal antibody, in patients with moderate-to-severe plaque psoriasis.

Methods Patients who completed a 12-week, phase II, dose-ranging clinical trial received brodalumab $210 \mathrm{mg}$ every 2 weeks in an open-label extension study. Efficacy was assessed by static physician's global assessment (sPGA) and psoriasis area and severity index (PASI). Quality of life, assessed by dermatology life quality index (DLQI), and safety were also evaluated.

Results Overall, 181 patients received brodalumab for a median of 264 weeks. Brodalumab treatment resulted in rapid improvements in SPGA, PASI, and DLQI that were maintained through week 264. Achieving PASI 90 to $<100$ or PASI 100 at weeks 12, 240, and 264 was associated with greater likelihood for DLQI 0 or 1 compared with achieving PASI 75 to $<90$. Over 5 years, one adverse event of suicidal ideation was reported, no suicides occurred, and no new safety signals emerged. Conclusions Brodalumab demonstrated skin clearance and improved quality of life, with an acceptable safety profile, throughout 5 years of treatment.
\end{abstract}

ClinicalTrials.gov Identifier NCT01101100.

\section{Introduction}

Psoriasis is a chronic inflammatory disease affecting $>7$ million adults in the United States, where prevalence estimates

Bausch Health Americas, Inc. and Bausch Health US, LLC are affiliates of Bausch Health Companies Inc; Ortho Dermatologics is a division of Bausch Health US, LLC.

Electronic supplementary material The online version of this article (https://doi.org/10.1007/s40257-019-00466-2) contains supplementary material, which is available to authorized users.

Mark G. Lebwohl

Lebwohl@aol.com

1 Icahn School of Medicine at Mount Sinai, 5 East 98th Street, 5th Floor, New York, NY 10029, USA

2 Oregon Medical Research Center, 9495 SW Locust Street, Suite G, Portland, OR 97223, USA

3 Baylor Scott \& White, 3900 Junius Street, Suite 145, Dallas, TX 75246, USA

4 Probity Medical Research and K. Papp Clinical Research, 135 Union Street East, Waterloo, ON N2J 1C4, Canada range from 1 to 3\% [1-3]. The burden of psoriasis may be higher in other regions of the world, with prevalence rates ranging from 1 to $8 \%$ in Europe and Australia [2]. Although psoriasis is primarily described in terms of its dermatologic manifestations, the disease itself has far-reaching implications for the patient, negatively affecting health-related quality of life and emotional well-being, increasing comorbidity and mortality risk and the likelihood of suicidal ideation, creating social stigma, and interfering with employment and work productivity [4-6]. Biologic therapies have been shown

5 The Dermatology Center of Indiana, 1100 Southfield Drive, Suite 1240, Plainfield, IN 46168, USA

6 Bausch Health Americas, 1330 Redwood Way \#A, Petaluma, CA 94954, USA

7 Bausch Health US, LLC, 400 Somerset Corporate Boulevard, Bridgewater, NJ 08807, USA

8 Ortho Dermatologics, 400 Somerset Corporate Boulevard, Bridgewater, NJ 08807, USA 


\section{Key Points}

Long-term use of brodalumab improved skin clearance and quality of life in patients with moderate-to-severe psoriasis.

High skin clearance scores were associated with a greater likelihood of improvement in quality of life.

Brodalumab is safe for long-term use.

to promote skin clearance $[7,8]$, which has been linked to improvement in quality of life [9, 10]. However, because psoriasis is a chronic condition, there is a need to demonstrate that this improvement is sustained during long-term treatment and that patients continue to tolerate and maintain treatment over the course of many years.

Brodalumab is a fully human anti-interleukin-17 receptor A monoclonal antibody that is efficacious for the treatment of moderate-to-severe plaque psoriasis. In pivotal clinical trials, brodalumab was superior to ustekinumab, a biologic therapy targeting interleukin-12 and interleukin-23, for skin clearance in patients with moderate-to-severe plaque psoriasis $[11,12]$. Phase II and III clinical trials have also shown improvements in health-related quality of life, depression, and anxiety among patients who received brodalumab [12-15].

Interim data from an open-label extension study of a phase II, dose-ranging clinical trial demonstrated that clinical response to brodalumab was maintained through 120 weeks and that brodalumab had an acceptable safety profile during long-term treatment [16]. The analysis reported herein expands on these results, describing the efficacy, safety, and patient-reported outcome findings over the course of 5 years of brodalumab treatment in patients with moderate-to-severe plaque psoriasis.

\section{Materials and Methods}

\subsection{Patients}

Patient eligibility criteria and study methodologies for the 12-week, phase II, double-blind, placebo-controlled, dose-ranging clinical trial (the parent study; ClinicalTrials.gov identifier: NCT00975637) and the open-label long-term extension study (ClinicalTrials.gov identifier: NCT01101100) have been previously described [13, 16]. Briefly, patients were 18-70 years of age at baseline of the parent study and had moderate-to-severe plaque psoriasis, defined as psoriasis-affected body surface area (BSA) of $\geq 10 \%$ and psoriasis area and severity index (PASI) of $\geq 12$. Patients who remained in the study were eligible for enrollment in the long-term extension study if they had completed an additional visit at week 16 of the parent study and had not experienced any serious adverse events (AEs) that were considered by the investigator to be related to study treatment $[13,16]$.

\subsection{Study Design}

In the parent study, patients were randomized $1: 1: 1: 1: 1$ to treatment with brodalumab $70,140,210$, or $280 \mathrm{mg}$ or placebo for 12 weeks [13]. At the baseline visit of the long-term extension study, patients who had received brodalumab in the parent study restarted treatment with brodalumab after a hiatus of $\geq 6$ weeks. Patients who had received placebo in the parent study initiated treatment with brodalumab for the first time. All patients in the long-term extension study received brodalumab $210 \mathrm{mg}$ subcutaneously at baseline, at weeks 1 and 2, and then every 2 weeks (Q2W) thereafter. A protocol amendment enacted at week 59 allowed patients weighing $\leq 100 \mathrm{~kg}$ to switch to brodalumab $140 \mathrm{mg} \mathrm{Q} 2 \mathrm{~W}$ (which is lower than the US Food and Drug Administration [FDA]-approved dose) [17]. A second amendment enacted at week 123 allowed patients with an inadequate response (defined as static physician's global assessment [sPGA] score of 2 for $\geq 4$ weeks or a single sPGA score of $\geq 3$ ) during treatment with brodalumab $140 \mathrm{mg} \mathrm{Q} 2 \mathrm{~W}$ to have their dose increased to $210 \mathrm{mg} \mathrm{Q} 2 \mathrm{~W}$ (the FDA-approved dose) [17]. Treatment was intended to continue to 360 weeks; however, the study was stopped early by the sponsor at that time for administrative reasons. When the study was terminated, 126 patients who remained in the study were withdrawn from the study. Data are reported herein to week 264 because all continuing patients had completed that visit.

\subsection{Assessments}

Study visits were conducted at weeks 1, 2, 4, 6, 8, 10, 12, $16,20,24,36$, and 48 and every 24 weeks thereafter. Efficacy assessments included the percentage of patients with an sPGA score of 0 (clear) or $\leq 1$ (clear or almost clear skin); the percentage of patients who experienced a $75 \%, 90 \%$, or $100 \%$ improvement from baseline in PASI (PASI 75, PASI 90, and PASI 100, respectively); the mean percent improvement in PASI; and the mean percent improvement in BSA involvement. Patient-reported outcome measures included the dermatology life quality index (DLQI) and the Medical Outcomes Study Short Form 36 health survey, version 2 (SF-36v2) mental and physical component scores. DLQI success was measured by the percentage of patients with a DLQI of 0 or 1 (DLQI 0/1). 
Safety was assessed by the occurrence of AEs and serious AEs as well as changes in vital signs and laboratory parameters. Laboratory results were graded using Common Terminology Criteria for Adverse Events, version 4.03. Adverse events of special interest, including neutropenia, infections, injection-site reactions, nervous system disorders, psychiatric disorders, and hypersensitivity, were also summarized.

\subsection{Statistical Analyses}

All patients who received at least one dose of brodalumab in the open-label extension study were included in efficacy and safety analyses. Data were analyzed using descriptive statistics, with no formal statistical testing. Summary statistics included the number, percentage, and 2-sided $95 \%$ confidence interval (CI) for categorical variables and the number of observations, mean, standard deviation (SD), and $95 \%$ CI for continuous variables. Incidence rates for AEs were reported as the percentage and as the exposureadjusted event rate per 100 patient-years. Data were analyzed as observed or with nonresponder imputation (NRI; for categorical variables) at each time point.

\section{Results}

A total of 181 patients who had completed the phase II, double-blind, placebo-controlled, dose-ranging parent study continued into the open-label extension study (Fig. 1). Of these 181 patients, 148 patients received brodalumab and 33 patients received placebo. Because the long-term extension study was stopped early for administrative reasons, no patients were considered to have completed the study. At the

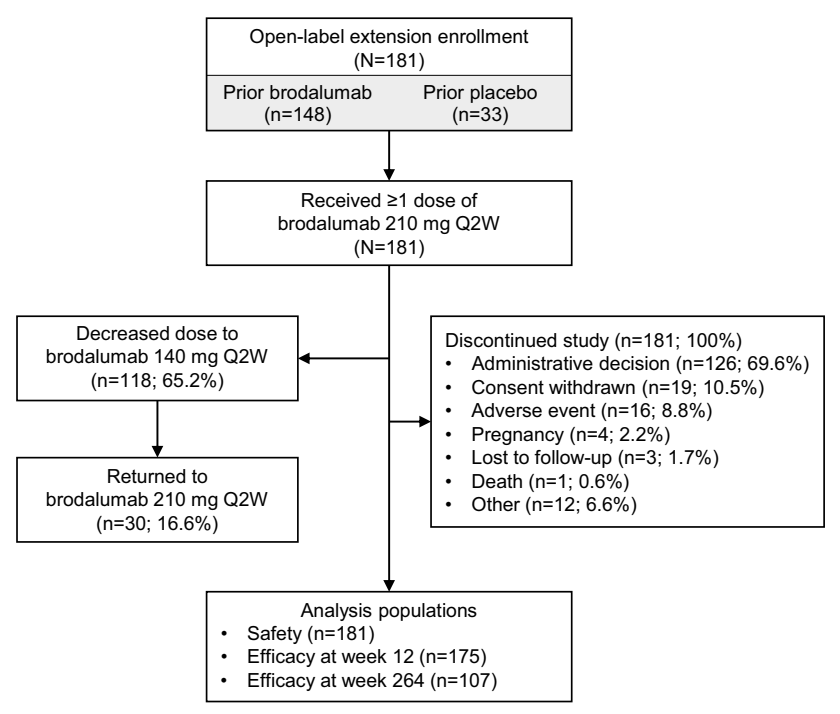

Fig. 1 Patient disposition. $Q 2 W$ every 2 weeks time the study was stopped, 123 patients $(68.0 \%)$ were still receiving brodalumab. All patients remaining in the study at the time it was discontinued had data available through week 264, which served as the final assessment time point in this analysis. By week 264, patients had not been receiving study treatment for $\geq 6$ weeks. Among 181 patients who entered the open-label study, 55 patients $(30.4 \%)$ discontinued study treatment; the most common reasons were consent withdrawal $(n=19 ; 10.5 \%)$ and $\mathrm{AE}(n=16 ; 8.8 \%)$.

Median duration of brodalumab exposure was 264 weeks (interquartile range 200-274 weeks). Dose reductions from brodalumab 210 to $140 \mathrm{mg}$ Q2W due to body weight of $\leq 100 \mathrm{~kg}$ occurred in 118 patients $(65.2 \%)$; 30 patients (16.6\%) subsequently had dose increases to brodalumab $210 \mathrm{mg}$ Q2W because of inadequate responses to brodalumab 140 mg Q2W.

Patient demographics and baseline characteristics have been previously reported [16]. Most patients were male $(64.6 \%)$, and the mean age at the start of the open-label extension study was 43.1 years. Disease severity (e.g., mean PASI, percent BSA involvement, and sPGA score) was not as severe at the start of the open-label extension study compared with that at baseline of the parent study, consistent with a large proportion of patients having received active treatment in the parent study. A total of 45 patients $(24.9 \%)$ had psoriatic arthritis, and the mean duration of psoriasis at baseline was 18.9 years (range $1-52$ years).

\subsection{Efficacy}

The proportion of patients who had an observed sPGA score of $\leq 1$ (clear or almost clear skin) was $63.0 \%$ (95\% CI 55.3-70.2) at week 2 and 90.3\% (95\% CI 84.9-94.2) at week $12 ; 77.3 \%$ (95\% CI $69.1-84.3)$ of patients maintained this level of response through week 240 (Fig. 2). The proportion of patients with an observed sPGA score of 0 followed a generally similar pattern. Using NRI analysis, the proportion of patients with an sPGA score of $\leq 1$ was $87.3 \%$ (95\% CI 81.5-91.8) at week 12, remained $>50 \%$ through week 240 , and was 36.5\% (95\% CI 29.5-43.9) at week 264 (Fig. E1 in the Electronic Supplementary Material [ESM]). There were modest decreases in responses with both SPGA scores of 0 and sPGA scores of $\leq 1$ when lower doses of brodalumab (140 mg Q2W) were introduced in patients weighing $\leq 100 \mathrm{~kg}$, and there was a return to pre-dose-reduction responses after reintroduction of increased doses (brodalumab $210 \mathrm{mg} \mathrm{Q} 2 \mathrm{~W}$ ) in patients demonstrating inadequate responses to lowered doses. Likewise, there were decreases in responses at week 264 (the final study visit) because patients had been off treatment for $\geq 6$ weeks.

There were similar results for PASI 75, PASI 90, and PASI 100 (Fig. 3). At week 8, observed PASI 75 response was $94.9 \%$ (95\% CI 90.5-97.6), with responses maintained 


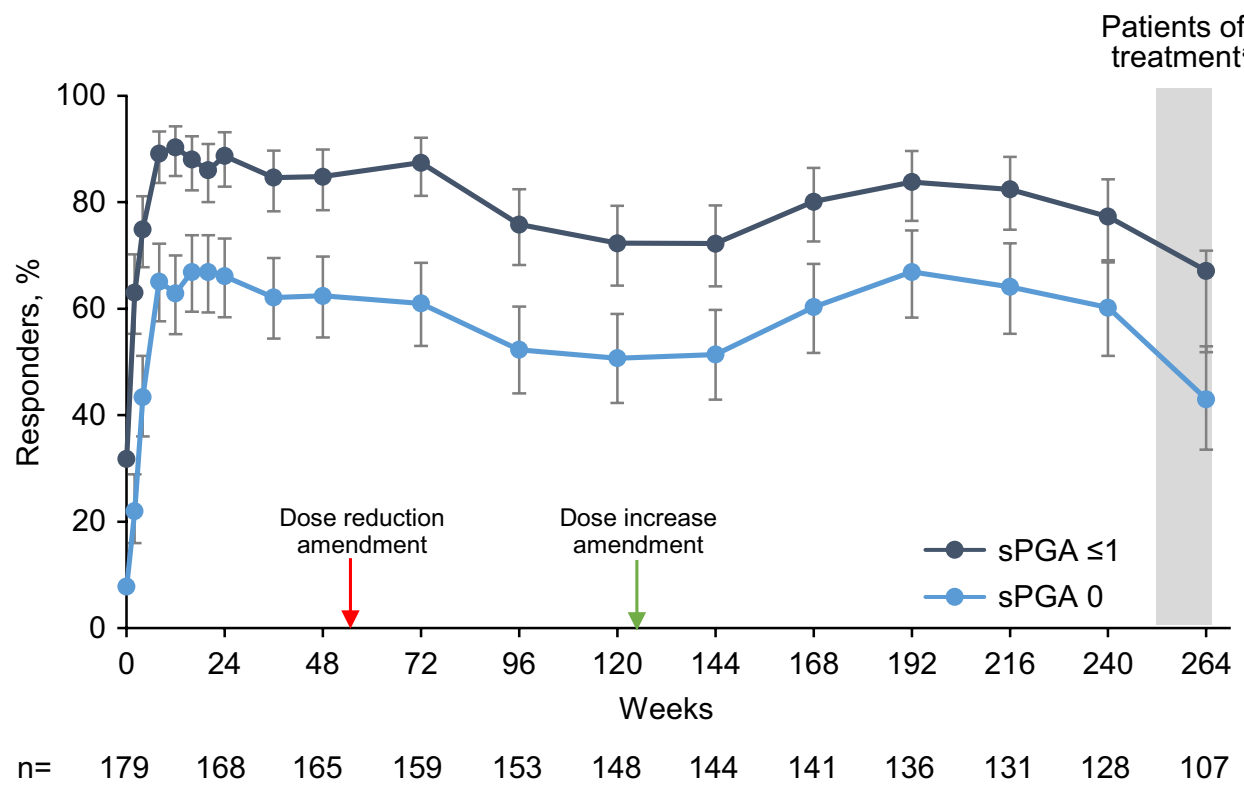

Fig. 2 Percentage of patients with sPGA score of 0 (clear) or $\leq 1$ (clear or almost clear) at each study visit. Observed data analysis. Error bars show the 95\% confidence interval. The red arrow indicates introduction of the protocol amendment that allowed dose reductions to brodalumab $140 \mathrm{mg}$ for patients weighing $\leq 100 \mathrm{~kg}$. The green arrow indicates introduction of the protocol amendment that allowed dose increases to brodalumab $210 \mathrm{mg}$ for patients demonstrating an insufficient response with the 140-mg dose. $n$ number of patients who had a valid measurement value at the specified week, $S P G A O$ and $s P G A \leq 1$ static physician's global assessment score of 0 and $\leq 1$. ${ }^{\text {a At }}$ week 264 , patients had been off treatment for $\geq 6$ weeks

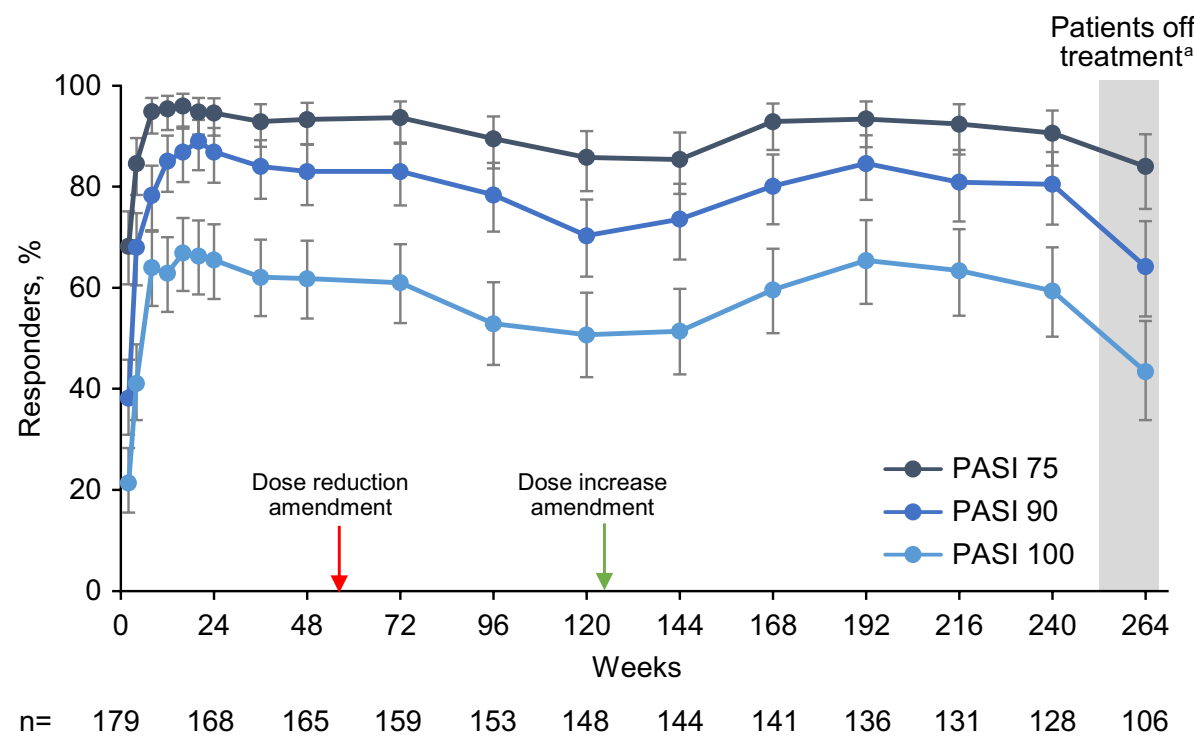

Fig. 3 Percentage of patients with skin clearance response measured by PASI at each study visit. Observed data analysis. Error bars show the $95 \%$ confidence interval. The red arrow indicates introduction of the protocol amendment that allowed dose reductions to brodalumab $140 \mathrm{mg}$ for patients weighing $\leq 100 \mathrm{~kg}$. The green arrow indicates introduction of the protocol amendment that allowed dose increases to brodalumab $210 \mathrm{mg}$ for patients demonstrating an insufficient response with the 140-mg dose. $n$ number of patients who had a valid measurement value at the specified week; PASI 75, 90, and 100 psoriasis area and severity index $75 \%, 90 \%$, and $100 \%$ improvement. ${ }^{a} \mathrm{At}$ week 264 , patients had been off treatment for $\geq 6$ weeks 
at $>80 \%$ for weeks $4-264$. PASI responses were numerically lower with NRI analysis. PASI 75 response was $91.7 \%$ at week 8 (95\% CI 86.7-95.3), was maintained at $>60 \%$ through week 240 , and was $49.2 \%$ (95\% CI 41.7-56.7) at week 264 (Fig. E2 in the ESM). Complete skin clearance, as indicated by observed PASI 100, was achieved by $64.0 \%$ (95\% CI 56.4-71.1) of patients at week 8 and remained $\geq 50 \%$ through week 240 . PASI 100 response (with NRI analysis) was $\geq 40 \%$ from weeks 8 to 240 and was $25.4 \%$ (95\% CI 19.2-32.4) at week 264 (Fig. E2 in the ESM). As with sPGA scores, decreases in responses were observed after dose reductions, with restoration of response rates following dose increases and subsequent declines in responses after discontinuation of study treatment (week 264).

The mean percent improvement in PASI was $95.4 \%$ (95\% CI 93.9-96.9) at week 12. Percent improvement remained at approximately $\geq 90 \%$ through week 240 , where the mean percent improvement in PASI was $92.1 \%$ (95\% CI 89.1-95.1). Mean percent improvement in BSA involvement was $94.5 \%$ (95\% CI 92.5-96.4) at week 12 and 92.8\% (95\% CI 89.5-96.1) at week 240. At week 264, mean percent improvement in PASI and BSA involvement both decreased slightly but remained $>85 \%$.

\subsection{Patient-Reported Outcomes}

DLQI responses were measured by the percentage of patients with DLQI 0/1. At baseline of the open-label extension, $28.3 \%$ of patients had DLQI 0/1; by week 12 (the first post-baseline assessment time point), $83.9 \%$ of patients had DLQI 0/1 (Fig. 4). DLQI 0/1 was reported by more than two-thirds of patients throughout the long-term extension. DLQI was also analyzed in relation to the degree of PASI response. Patients with higher PASI response levels (PASI 90 to $<100$ or PASI 100) were more likely to have DLQI 0/1 compared with patients achieving PASI 75 to $<90$ (Fig. 5). These observations were consistent at weeks 12 and 240, and notably at week 264 , where patients had been off treatment for $\geq 6$ weeks.

At week 264, mean (SD) SF-36v2 mental component score increased relative to baseline of the open-label extension (baseline: 49.1 [11.0]; week 264: 55.5 [7.1]), with improvements maintained through week 264. Mean SF-36v2 physical component score did not change appreciably during the study.

\subsection{Safety}

Treatment-emergent AEs occurred in 177 patients (97.8\%). Grade $\geq 3$ AEs were reported in 41 patients (22.7\%), with 29 patients (16.0\%) experiencing one or more serious AE. The only serious AE reported by more than one patient was myocardial infarction (3 patients; $1.7 \%$ ). Twenty-one patients (11.6\%) discontinued study treatment because of an $\mathrm{AE}$, and 16 patients (8.8\%) withdrew from the study because of an AE. Treatment-emergent AEs leading to

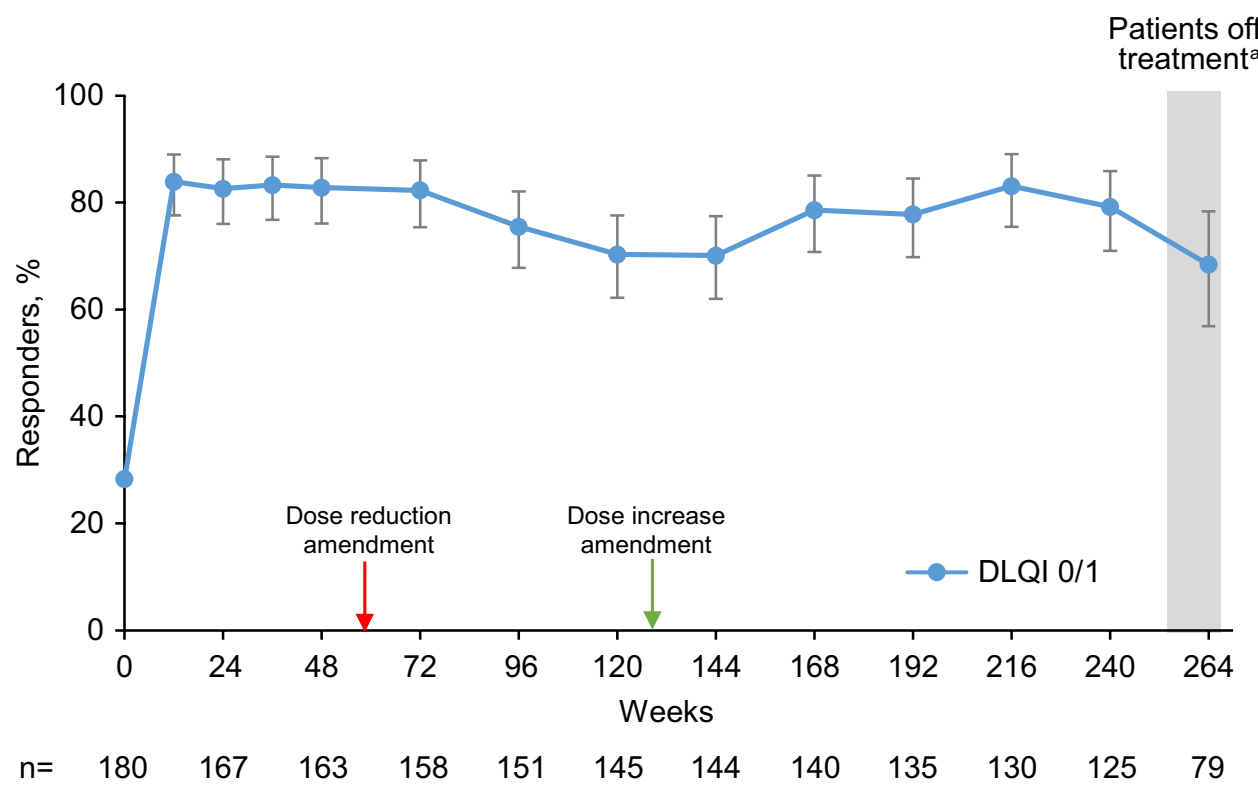

Fig. 4 Percentage of patients with DLQI 0/1 at each study visit. Observed data analysis. Error bars show the $95 \%$ confidence interval. The red arrow indicates introduction of the protocol amendment that allowed dose reductions to brodalumab $140 \mathrm{mg}$ for patients weighing $\leq 100 \mathrm{~kg}$. The green arrow indicates introduction of the protocol amendment that allowed dose increases to brodalumab $210 \mathrm{mg}$ for patients demonstrating an insufficient response with the 140-mg dose. $D L Q I 0 / 1$ dermatology life quality index 0 or $1, n$ number of patients who had a valid measurement value at the specified week. ${ }^{\text {a At week }}$ 264 , patients had been off treatment for $\geq 6$ weeks 
Fig. 5 Percentage of patients with DLQI 0/1 by skin clearance response, as measured by categories of PASI improvement from baseline (PASI 75 to $<90$, PASI 90 to $<100$, and PASI 100), at weeks 12, 240, and 264. Observed data analysis. Error bars show the $95 \%$ confidence interval. DLQI 0/1 dermatology life quality index 0 or 1, $n$ number of patients who had a valid measurement value at the specified week, PASI 75 , 90 , and 100 psoriasis area and severity index $75 \%, 90 \%$, and $100 \%$ improvement. ${ }^{\mathrm{a}}$ At week 264 , patients had been off treatment for $\geq 6$ weeks

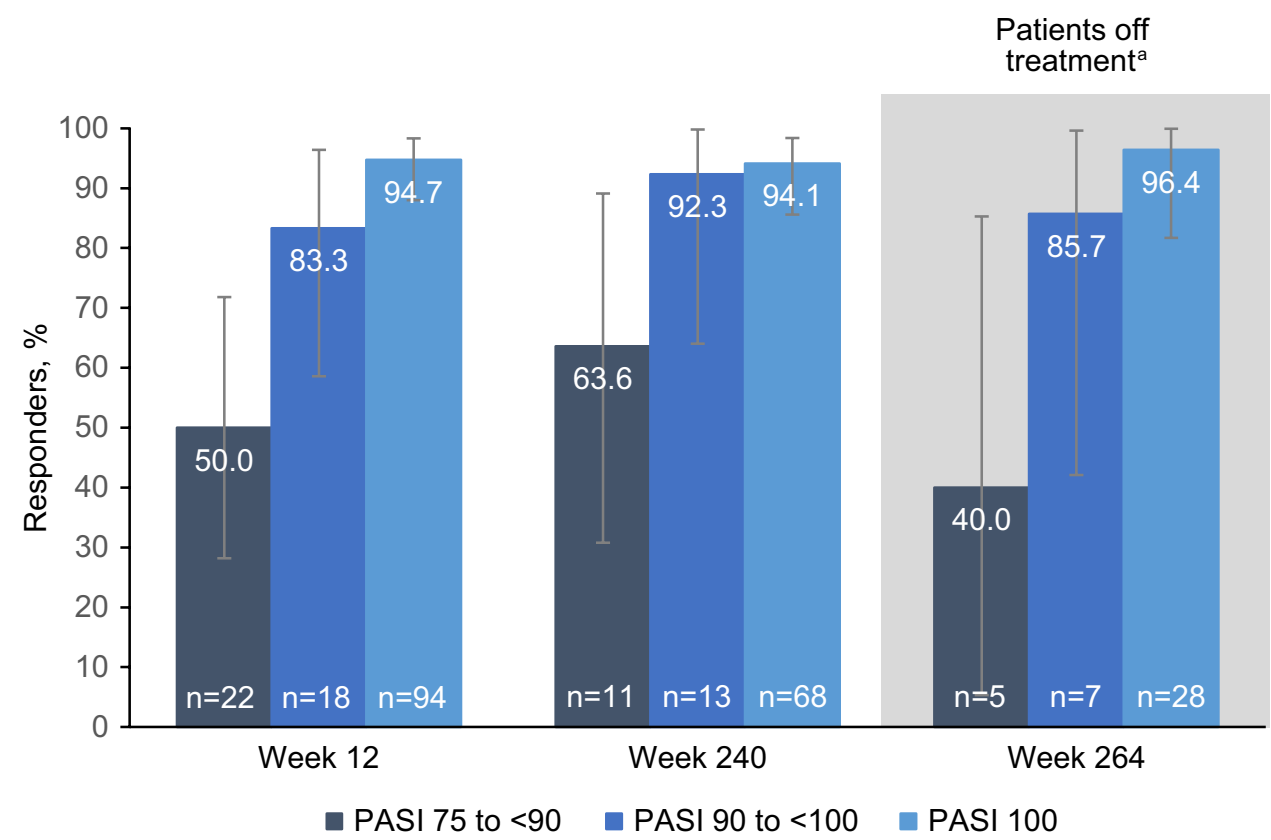

study discontinuation included six cases of worsening or flare of psoriasis, two neoplasms (esophageal adenocarcinoma and prostate cancer), one flare of guttate psoriasis, and one case each of psoriatic arthritis, pruritus, hepatitis $\mathrm{C}$, pyelonephritis, iridocyclitis, latent tuberculosis, and confusion. One patient experienced a fatal AE (aortic aneurysm rupture); this event was not considered by the investigator to be related to study treatment.

The overall exposure-adjusted AE rate was 278.1 per 100 patient-years, which generally decreased over time (Table 1). The most common AEs included nasopharyngitis, upper respiratory tract infection, and arthralgia (29\%, 24\%, and $20 \%$, respectively). AEs of special interest, such as nervous system disorders, injection-site reactions, psychiatric disorders, and oropharyngeal candidiasis, occurred at exposureadjusted event rates of $<17$ per 100 patient-years. Across all study years, Candida infection and Staphylococcus infection each occurred in $0.6 \%$ of patients, and psoriatic arthropathy occurred in $10 \%$. No suicides occurred, and one AE of suicidal ideation was reported over the 5-year treatment period (exposure-adjusted rate 0.1 per 100 patient-years).

\section{Discussion}

Many factors should be considered when selecting an appropriate therapy for moderate-to-severe psoriasis [18, 19]. Speed of onset and durability of response are among those factors [20]. In this open-label extension study, treatment with brodalumab in patients with moderate-to-severe plaque psoriasis resulted in substantial improvement from baseline in skin clearance and health-related quality of life that was sustained over 5 years. A number of patients achieved clear or almost clear skin within the first 2 weeks of treatment, with further improvements occurring through week 12. Maintenance of efficacy was observed through week 264, when patients had been off treatment for $\geq 6$ weeks. These efficacy results are notable given that $16.6 \%$ of patients had dose increases to brodalumab $210 \mathrm{mg}$ Q2W because of inadequate responses to brodalumab $140 \mathrm{mg} \mathrm{Q} 2 \mathrm{~W}$ (which is lower than the FDA-approved dose) [21]. Further, brodalumab was associated with long-term maintenance of skin clearance efficacy in a phase III study [22].

This study also demonstrated that the AE profile of longterm brodalumab treatment is consistent with that observed in shorter-term studies, and that extended treatment over 5 years was not associated with any new safety signals $[13,16]$. Importantly, no suicides occurred, and one AE of suicidal ideation was reported over the 5-year treatment period of the phase II study. In a separate group of patients in the phase III psoriasis studies, three confirmed suicides occurred late in the study program, but there has been no causal association between brodalumab and suicidal ideation and behavior established [23].

Improvements in quality of life as measured by the DLQI were pronounced during this study. Greater than two-thirds of patients achieved DLQI 0/1 from week 12 through week 264. On the basis of the current analysis discussed in this article, there was an association between skin clearance and DLQI, such that patients with PASI 90 to $<100$ or PASI 100 were more likely to have DLQI 0/1 compared with patients with PASI 75 to $<90$. Similar relationships between skin clearance and quality-of-life measures have been reported in the literature [9, 10, 24, 25]. Mattei et al. 
Table 1 Exposure-adjusted event rates of TEAEs by year among all patients who received any dose of brodalumab

\begin{tabular}{lllllll}
\hline & Year 1 $(n=181)$ & Year 2 $(n=181)$ & Year 3 $(n=181)$ & Year 4 $(n=181)$ & Year 5 $(n=181)$ & Year 6 $(n=181)$ \\
\hline Total patient-years of exposure & 172.9 & 330.1 & 475.2 & 611.0 & 725.7 & 731.7 \\
Number of TEAEs (exposure-adjusted event rate per 100 patient-years) & & & \\
All TEAEs & $748(432.6)$ & $1154(349.6)$ & $1373(288.9)$ & $1565(256.1)$ & $1750(241.1)$ & $1770(241.9)$ \\
Grade $\geq 2$ & $380(219.8)$ & $593(179.6)$ & $733(154.3)$ & $863(141.2)$ & $997(137.4)$ & $1014(138.6)$ \\
Grade $\geq 3$ & $18(10.4)$ & $29(8.8)$ & $37(7.8)$ & $49(8.0)$ & $59(8.1)$ & $62(8.5)$ \\
Serious TEAEs & $13(7.5)$ & $18(5.5)$ & $22(4.6)$ & $31(5.1)$ & $40(5.5)$ & $41(5.6)$ \\
TEAEs of interest (exposure-adjusted event rate per 100 patient-years) & & & \\
Nervous system disorder & $29(16.8)$ & $51(15.4)$ & $62(13.0)$ & $78(12.8)$ & $90(12.4)$ & $91(12.4)$ \\
Injection-site reaction & $28(16.2)$ & $38(11.5)$ & $40(8.4)$ & $40(6.5)$ & $41(5.6)$ & $41(5.6)$ \\
Psychiatric disorder & $19(11.0)$ & $27(8.2)$ & $32(6.7)$ & $44(7.2)$ & $49(6.8)$ & $49(6.7)$ \\
Oropharyngeal candidiasis & $17(9.8)$ & $23(7.0)$ & $28(5.9)$ & $34(5.6)$ & $41(5.6)$ & $41(5.6)$ \\
Hypersensitivity event & $8(4.6)$ & $12(3.6)$ & $12(2.5)$ & $12(2.0)$ & $12(1.7)$ & $12(1.6)$ \\
Depression & $4(2.3)$ & $4(1.2)$ & $6(1.3)$ & $7(1.1)$ & $9(1.2)$ & $9(1.2)$ \\
Serious infectious episode & $4(2.3)$ & $6(1.8)$ & $6(1.3)$ & $7(1.1)$ & $8(1.1)$ & $8(1.1)$ \\
Ischemic heart disease & 0 & 0 & $1(0.2)$ & $4(0.7)$ & $7(1.0)$ & $7(1.0)$ \\
Neutropenia & $1(0.6)$ & $1(0.3)$ & $1(0.2)$ & $1(0.2)$ & $1(0.1)$ & $1(0.1)$ \\
Suicide self-injury & 0 & 0 & 0 & 0 & 0 & $1(0.1)$
\end{tabular}

The total patient-years of exposure and number of TEAEs and TEAEs of interest are cumulative

TEAE treatment-emergent adverse event

${ }^{a}$ Multiple occurrences of the same event for a patient are counted as multiple events. Exposure-adjusted event rate per 100 patient-years calculated as number of events/total patient-years of exposure $\times 100$

${ }^{\mathrm{b}}$ Indicates a TEAE of suicidal ideation

reported a correlation $\left(r^{2}=0.80\right)$ between percentage of PASI improvement from baseline and changes in DLQI among patients with moderate-to-severe psoriasis using data from 13 randomized controlled trials of biologic therapies [9]. Similar to the findings in our study, a meta-analysis by Puig et al. detected a greater improvement in DLQI among patients with PASI 90 versus patients with PASI 75-89 [24]. Although Abrouk et al. reported inconsistent differentiation of DLQI in patients achieving PASI 90 versus those achieving PASI 75 [26], the preponderance of evidence highlights the effect that achieving clear or almost clear skin has on improving the quality of life in patients with moderate-tosevere psoriasis.

A notable finding from this study was the considerable number of patients $(68.0 \%)$ who continued to receive brodalumab at the time the study was stopped for administrative reasons. Drug survival (or persistence with therapy) is a known challenge with biologic therapies. In the prospective study of the British Association of Dermatologists Biologic Interventions Register (BADBIR), overall drug survival rates for a first course of biologic therapy among 3523 biologic-naive patients were $77 \%$ for the first year, $63 \%$ for the second year, and $53 \%$ for the third year [27]. Similar results were reported for 1239 patients enrolled in the BADBIR who had inadequate responses to first-line biologic therapy and switched to second-line biologic agents [28]. Among $\sim 3500$ patients evaluated in an analysis of the Psoriasis Longitudinal Assessment and Registry (PSOLAR), median duration of treatment before stopping or switching biologic therapy was 2.5 years for first-line therapy and 3.2 years for second-line therapy [29]. Most patients discontinued therapy because of lack of effectiveness. Heterogeneity of drug survival rates for individual biologic therapies was observed in both registries [27-29]. Brodalumab was not included in the available registry data because it was approved only after these analyses were performed. When comparing BADBIR and PSOLAR data with our study observations, the differences between registry and clinical trial participants should be considered. Patients enrolled in a clinical study may reflect a healthier population that has a lower risk of AEs and, hence, may be better able to sustain therapy. However, using data from BADBIR, investigators found no significant differences in discontinuation rates between patients who would be deemed eligible and those who would be deemed ineligible for participation in clinical trials [30]. The suggestion of a potential benefit in terms of drug survival for brodalumab merits further investigation using real-world patient data. 
Because this open-label extension study did not include a comparator arm, there are limitations inherent to the study design, including potential selection bias for patients who may be more likely to tolerate or respond to the study treatment [16]. Consistency of the phase II long-term extension study data with that of the maintenance phase of a phase III, 52-week, randomized controlled study of brodalumab [11] bolsters the finding that brodalumab is associated with longterm efficacy. Sustained clinical response was also observed in a 52-week open-label extension study of brodalumab in Japanese patients with moderate-to-severe plaque psoriasis [31]. Other limitations included small sample size and early termination of the study.

\section{Conclusion}

Overall, long-term therapy with brodalumab in this study resulted in sustained improvements in skin clearance and health-related quality of life, with a safety profile comparable to that of shorter-term treatment. These data add evidence that supports brodalumab as an efficacious and well-tolerated biologic therapy for the long-term treatment of moderate-to-severe plaque psoriasis, a lifelong disease.

Acknowledgements We gratefully acknowledge Kim A. Papp as the coordinating investigator of this phase II program, including the longterm extension. Medical writing support was provided by Crystal Murcia, PhD, Rebecca E. Slager, PhD, and David Boffa, ELS, of MedThink SciCom, under the direction of the authors, and was funded by Ortho Dermatologics. Ortho Dermatologics is a division of Bausch Health US, LLC.

Data Availability The datasets generated and/or analyzed during the current study are available from the corresponding author on reasonable request.

\section{Compliance with Ethical Standards}

Funding The brodalumab clinical study program was sponsored by Amgen/AstraZeneca, and this analysis was performed by Amgen/ AstraZeneca.

Conflict of interest Mark G. Lebwohl is an employee of Mount Sinai, which receives research funds from AbbVie, Boehringer Ingelheim, Bausch Health, Celgene, Eli Lilly, Incyte, Janssen/Johnson \& Johnson, Leo, Medimmune/Astra Zeneca, Novartis, Pfizer, Sciderm, $\mathrm{UCB}$, and Vidac, and is also a consultant for Allergan, Aqua, Arcutis, Boehringer-Ingelheim, Leo, Menlo, and Promius. Andrew Blauvelt has served as a scientific adviser and/or clinical study investigator for AbbVie, Aclaris, Akros, Allergan, Almirall, Amgen, Arena, Athenex, Boehringer Ingelheim, Bristol-Myers Squibb, Celgene, Dermavant, Dermira, Eli Lilly, FLX Bio, Galderma, Genentech/Roche, GlaxoSmithKline, Janssen, Leo, Meiji, Merck Sharp \& Dohme, Novartis, Pfizer, Purdue Pharma, Regeneron, Revance, Sandoz, Sanofi Genzyme, Sienna Pharmaceuticals, Sun Pharma, UCB, and Vidac and as a paid speaker for AbbVie, Regeneron, and Sanofi Genzyme. Alan Menter has received compensation from or served as an investigator, consultant, advisory board member, or speaker for AbbVie, Allergan, Amgen, Anacor, Boehringer Ingelheim, Celgene, Dermira, Eli Lilly, Galderma, Janssen, Leo, Merck \& Co, Neothetics, Novartis, Pfizer, Regeneron, Symbio/Maruho, Vitae, and Xenoport. Kim A. Papp has served as a consultant; scientific officer; member of a speaker's bureau, advisory board, or steering committee for or received research grants or honoraria from AbbVie, Akesis, Akros, Allergan, Alza, Amgen, Anacor, Artax Biopharma, Astellas, AstraZeneca, Bausch Health, Baxter, Baxalta, Boehringer Ingelheim, Bristol-Myers Squibb, Can-Fite BioPharma, Celgene, Celtic Pharma, Cipher, Dermira, Dow Pharma, Eli Lilly, Ferring, Formycon, Forward Pharma, Fujisawa, Funxional Therapeutics, Galderma, Genentech, Genexion, Genzyme, Gilead, GlaxoSmithKline, Janssen, Kyowa Hakko Kirin Co, Leo, MedImmune, Meiji Seika Pharma Co, Merck \& Co (MSD), Merck Serono, Mitsubishi Tanabe Pharma, Mylan, Novartis, NovImmune, Pan-Genetics, Pfizer, Regeneron, Roche, Sanofi-Aventis, Stiefel Laboratories, Takeda, UCB, and Vertex Pharmaceuticals. Scott Guenthner serves as a speaker for Janssen, Pfizer, AbbVie, Sun Pharma, and Aclaris. Radhakrishnan Pillai is an employee of Bausch Health Americas and may hold stock and/or stock options in the company. Robert J. Israel is an employee of Bausch Health US, LLC and holds stock and/or stock options in the company. Abby Jacobson is an employee of Ortho Dermatologics and holds stocks and/or stock options in Bausch Health US, LLC.

Ethical standards The study protocol received institutional review board or ethics committee approval at each participating site, and study procedures were conducted in accordance with the ethical principles established by the Declaration of Helsinki. Informed consent was obtained from all individual participants included in the study.

Open Access This article is distributed under the terms of the Creative Commons Attribution-NonCommercial 4.0 International License (http://creativecommons.org/licenses/by-nc/4.0/), which permits any noncommercial use, distribution, and reproduction in any medium, provided you give appropriate credit to the original author(s) and the source, provide a link to the Creative Commons license, and indicate if changes were made.

\section{References}

1. Rachakonda TD, Schupp CW, Armstrong AW. Psoriasis prevalence among adults in the United States. J Am Acad Dermatol. 2014;70:512-6.

2. Parisi R, Symmons DP, Griffiths CE, Ashcroft DM. Global epidemiology of psoriasis: a systematic review of incidence and prevalence. J Invest Dermatol. 2013;133:377-85.

3. Lebwohl MG, Bachelez H, Barker J, Girolomoni G, Kavanaugh A, Langley RG, et al. Patient perspectives in the management of psoriasis: results from the population-based Multinational Assessment of Psoriasis and Psoriatic Arthritis Survey. J Am Acad Dermatol. 2014;70(871-81):e1-30.

4. Armstrong AW, Schupp C, Wu J, Bebo B. Quality of life and work productivity impairment among psoriasis patients: findings from the National Psoriasis Foundation survey data 2003-2011. PLoS One. 2012;7:e52935.

5. Feldman SR, Goffe B, Rice G, Mitchell M, Kaur M, Robertson $\mathrm{D}$, et al. The challenge of managing psoriasis: unmet medical needs and stakeholder perspectives. Am Health Drug Benefits. 2016;9:504-13.

6. Strober B, Karki C, Mason M, Guo N, Holmgren SH, Greenberg JD, et al. Characterization of disease burden, comorbidities, and treatment use in a large, US-based cohort: results from the Corrona Psoriasis Registry. J Am Acad Dermatol. 2018;78:323-32. 
7. Sbidian E, Chaimani A, Garcia-Doval I, Do G, Hua C, Mazaud $\mathrm{C}$, et al. Systemic pharmacological treatments for chronic plaque psoriasis: a network meta-analysis. Cochrane Database Syst Rev. 2017;12:CD011535.

8. Sawyer L, Fotheringham I, Wright E, Yasmeen N, Gibbons C, Holmen Moller A. The comparative efficacy of brodalumab in patients with moderate-to-severe psoriasis: a systematic literature review and network meta-analysis. J Dermatolog Treat. 2018;29:557-68.

9. Mattei PL, Corey KC, Kimball AB. Psoriasis area severity index (PASI) and the dermatology life quality index (DLQI): the correlation between disease severity and psychological burden in patients treated with biological therapies. J Eur Acad Dermatol Venereol. 2014;28:333-7.

10. Elewski BE, Puig L, Mordin M, Gilloteau I, Sherif B, Fox T, et al. Psoriasis patients with psoriasis area and severity index (PASI) 90 response achieve greater health-related quality-oflife improvements than those with PASI 75-89 response: results from two phase 3 studies of secukinumab. J Dermatolog Treat. 2017;28:492-9.

11. Lebwohl M, Strober B, Menter A, Gordon K, Weglowska J, Puig $\mathrm{L}$, et al. Phase 3 studies comparing brodalumab with ustekinumab in psoriasis. N Engl J Med. 2015;373:1318-28.

12. Papp KA, Reich K, Paul C, Blauvelt A, Baran W, Bolduc C, et al. A prospective phase III, randomized, double-blind, placebo-controlled study of brodalumab in patients with moderate-to-severe plaque psoriasis. Br J Dermatol. 2016;175:273-86.

13. Papp KA, Leonardi C, Menter A, Ortonne JP, Krueger JG, Kricorian G, et al. Brodalumab, an anti-interleukin-17-receptor antibody for psoriasis. N Engl J Med. 2012;366:1181-9.

14. Gordon KB, Kimball AB, Chau D, Viswanathan HN, Li J, Revicki DA, et al. Impact of brodalumab treatment on psoriasis symptoms and health-related quality of life: use of a novel patient-reported outcome measure, the psoriasis symptom inventory. Br J Dermatol. 2014;170:705-15.

15. Nakagawa $H$, Niiro $H$, Ootaki K. Brodalumab, a human anti-interleukin-17-receptor antibody in the treatment of Japanese patients with moderate-to-severe plaque psoriasis: efficacy and safety results from a phase II randomized controlled study. J Dermatol Sci. 2016;81:44-52.

16. Papp K, Leonardi C, Menter A, Thompson EH, Milmont CE, Kricorian G, et al. Safety and efficacy of brodalumab for psoriasis after 120 weeks of treatment. J Am Acad Dermatol. 2014;71(1183-90):e3.

17. Papp K BH, Iversen L, Rosen M, Hansen K, Menter A. Complete clearance is sustained during long-term ( $\sim 5$ years) treatment with brodalumab in moderate-to-severe psoriasis. 26th EADV Congress; Geneva, Switzerland; 2017.

18. Kaushik SB, Lebwohl MG. Psoriasis: which therapy for which patient: focus on special populations and chronic infections. J Am Acad Dermatol. 2019;80:43-53.

19. Kaushik SB, Lebwohl MG. Psoriasis: which therapy for which patient: Psoriasis comorbidities and preferred systemic agents. J Am Acad Dermatol. 2019;80:27-40.
20. Armstrong AW, Gordon KB, Menter MA, Wu JJ. The evolving landscape of psoriasis treatment. Semin Cutan Med Surg. 2018;37:S39-43.

21. Siliq ${ }^{\mathrm{TM}}$ (brodalumab) injection, for subcutaneous use Initial U.S. Approval. 2017. https://www.accessdata.fda.gov/drugsatfda_docs/ label/2017/761032lbl.pdf. Accessed 19 Aug 2019.

22. Puig L, Lebwohl MG, Bachelez H, Sobell J, Jacobson A. Longterm efficacy and safety of brodalumab in the treatment of psoriasis: 120 -week results from the randomized, double-blind, placebo- and active comparator-controlled phase 3 AMAGINE-2 trial. J Am Acad Dermatol. 2019. https://doi.org/10.1016/j. jaad.2019.05.095.

23. Lebwohl MG, Papp KA, Marangell LB, Koo J, Blauvelt A, Gooderham $\mathrm{M}$, et al. Psychiatric adverse events during treatment with brodalumab: analysis of psoriasis clinical trials. J Am Acad Dermatol. 2018;78(81-9):e5.

24. Puig L, Thom H, Mollon P, Tian H, Ramakrishna GS. Clear or almost clear skin improves the quality of life in patients with moderate-to-severe psoriasis: a systematic review and meta-analysis. J Eur Acad Dermatol Venereol. 2017;31:213-20.

25. Sojevic Timotijevic Z, Majcan P, Trajkovic G, Relic M, Novakovic $\mathrm{T}$, Mirkovic M, et al. The impact of changes in psoriasis area and severity index by body region on quality of life in patients with psoriasis. Acta Dermatovenerol Croat. 2017;25:215-22.

26. Abrouk M, Nakamura M, Zhu TH, Farahnik B, Koo J, Bhutani T. The impact of PASI 75 and PASI 90 on quality of life in moderate to severe psoriasis patients. J Dermatolog Treat. 2017;28:488-91.

27. Warren RB, Smith CH, Yiu ZZN, Ashcroft DM, Barker J, Burden $\mathrm{AD}$, et al. Differential drug survival of biologic therapies for the treatment of psoriasis: a prospective observational cohort study from the British Association of Dermatologists Biologic Interventions Register (BADBIR). J Invest Dermatol. 2015;135:2632-40.

28. Iskandar IYK, Warren RB, Lunt M, Mason KJ, Evans I, McElhone $\mathrm{K}$, et al. Differential drug survival of second-line biologic therapies in patients with psoriasis: observational cohort study from the British Association of Dermatologists Biologic Interventions Register (BADBIR). J Invest Dermatol. 2018;138:775-84.

29. Menter A, Papp KA, Gooderham M, Pariser DM, Augustin M, Kerdel FA, et al. Drug survival of biologic therapy in a large, disease-based registry of patients with psoriasis: results from the Psoriasis Longitudinal Assessment and Registry (PSOLAR). J Eur Acad Dermatol Venereol. 2016;30:1148-58.

30. Mason KJ, Barker J, Smith CH, Hampton PJ, Lunt M, McElhone $\mathrm{K}$, et al. Comparison of drug discontinuation, effectiveness, and safety between clinical trial eligible and ineligible patients in BADBIR. JAMA Dermatol. 2018;154:581-8.

31. Umezawa Y, Nakagawa H, Niiro H, Ootaki K. Long-term clinical safety and efficacy of brodalumab in the treatment of Japanese patients with moderate-to-severe plaque psoriasis. J Eur Acad Dermatol Venereol. 2016;30:1957-60. 